\title{
Radical of weakly ordered semigroup algebras
}

\author{
M. Schocker
}

Received: 16 January 2008 / Accepted: 31 January 2008 / Published online: 26 February 2008

(C) Springer Science+Business Media, LLC 2008

\begin{abstract}
We define the notion of weakly ordered semigroups. For this class of semigroups, we compute the radical of the semigroup algebras. This generalizes some results on left regular bands and on 0 - Hecke algebras.
\end{abstract}

Keywords Weak order $\cdot$ Left regular band $\cdot$ Semigroup algebra $\cdot$ Radical $\cdot$ Hecke algebra

\section{Foreword}

In the summer of 2006 Manfred and I projected to work on generalizing the constructions of [1] on left regular bands to more general semigroups. We wanted to define a class of semigroups for which we could compute the radical and the minimal idempotents of the semigroup algebras explicitly. Our hope was that this could be done uniformly for left regular bands (possibly even for bands in general) and for the 0Hecke algebras of finite types. Soon after, Manfred sent me the results presented here as the starting point of our project. I have added only the examples and the remarks showing how we intended to apply this result. — Nantel Bergeron (Toronto, 2007)

\section{Weakly ordered semigroups}

Let $\mathrm{S}$ be a finite semigroup with unity. If $u, v \in S$, we write $u \leq v$ if there exists an element $w \in S$ such that $u w=v$. This "weak ordering" on $\mathrm{S}$ is easily seen to be reflexive and transitive. However, in general it is not anti-symmetric. We write $u \sim v$ if $u \leq v$ and $v \leq u$.

M. Schocker (凶)

York University, 4700 Keele St., Toronto, M3J1P3, Canada

e-mail: Bergeron@mathstat.yorku.ca 
Let $(L, \preceq)$ be an upper semi-lattice. That is, a partially ordered set in which any two elements $I, J$ have a least upper bound, or join, $I \vee J$. The join operation turns L into a commutative semigroup.

Suppose there exist two maps $C: S \rightarrow L$ and $D: S \rightarrow L$ such that the following three conditions hold:

(1) The map $C$ is an epimorphism of semigroups.

(2) If $u, v \in S$ are such that $u v \sim u$, then $C(v) \preceq D(u)$.

(3) If $u, v \in S$ are such that $C(v) \preceq D(u)$, then $u v=u$.

Lemma 2.1 The conditions (2) and (3) above imply that $\leq$ is anti-symmetric, hence a partial order on $S$.

Proof Suppose $u, w \in S$ such that $u \sim w$. Then $u \leq w$, so we may choose $v \in S$ such that $w=u v$. Hence $u \sim u v$, and from (2) and (3) it follows that $u=u v=w$.

Definition 2.2 We say that a semigroup $S$ (finite with unity) is a weakly ordered semigroup if there exist two maps $C, D: S \rightarrow L$ satisfying the three conditions (1), (2) and (3).

If $I \in L$, let $S_{I}$ denote the set of all $v \in S$ such that $C(v)=I$. As usual, we write $u S_{I}=\left\{u v: v \in S_{I}\right\}$.

Lemma 2.3 Conditions (2) and (3) above imply that if we have $u \in S$ and $I \in L$ such that $u \in u S_{I}$, then $u S_{I}=\{u\}$.

Proof Let $u, v \in S$ and $I \in L$ such that $C(v)=I$ and $u=u v$. Then (2) implies that $I=C(v) \preceq D(u)$, hence $C\left(v^{\prime}\right) \preceq D(u)$ for all $v^{\prime} \in S_{I}$. By (3), it follows that $u v^{\prime}=u$ for all $v^{\prime} \in S_{I}$.

There are two main examples of weakly ordered semigroups.

Example 2.4 A semigroup $S$ is called a left regular band (see [1]) if there exists a lattice $L$ and a surjective map $C: S \rightarrow L$ such that $C(x y)=C(x) \vee C(y)$ and $x y=x$ whenever $C(y) \preceq C(x)$. The map $C$ in this context is referred to as the support map (or content map). This is equivalent the requirement that $x^{2}=x$ for all $x \in S$ and $x y x=x y$ for all $x, y \in S$. If we set $D=C$ then it is easy to check that such a semigroup $S$ is a weakly ordered semigroup.

Example 2.5 For a positive integer $n$, consider the semigroup $S$ generated by the elements $T_{1}, T_{2}, \ldots, T_{n}$ satisfying the relation $T_{i}^{2}=T_{i}$ and the braid relations $T_{i} T_{j}=$ $T_{j} T_{i}$ if $|i-j|>1$ and $T_{i} T_{i+1} T_{i}=T_{i+1} T_{i} T_{i+1}$. This is a presentation of $H_{n+1}(q)$ the Hecke algebra of type $A_{n}$ at $q=0$ (see [3]). Let $L$ be the lattice of subsets of $\{1,2, \ldots, n\}$. These relations imply that the map $C: S \rightarrow L$ where $C\left(T_{i_{1}} T_{i_{2}} \cdots T_{i_{\ell}}\right)=$ $\left\{i_{1}, i_{2}, \ldots, i_{\ell}\right\}$ is well defined. We also define $D: S \rightarrow L$ by $D(x)=\left\{i: x T_{i}=x\right\}$. We say that $C(x)$ is the content of $x$ and $D(x)$ is the descent set of $x$ (analogous to the descent set definition for Coxeter groups). Conditions (1) and (3) for these two 
maps are clear. To see that (2) holds as well, let $u v \sim u$. There is an $x \in S$ such that $u v x=u$. The length $\ell(u)=\ell$ of $u$ is defined by the length of a minimal expression for $u=T_{i_{1}} T_{i_{2}} \cdots T_{i_{\ell}}$. From the defining relations, $\ell\left(u T_{i}\right) \geq \ell(u)$ and $\ell\left(u T_{i}\right)=\ell(u)$ if and only if $i \in D(u)$. If $T_{i} \in C(v)$ but $T_{i} \notin D(u)$, then $\ell(u v x)>\ell(u)$ which contradicts the fact that $u v x=u$. So we must have $C(v) \subseteq D(u)$. Hence $S$ is a weakly ordered semigroup.

Remark that there is a weakly ordered semigroup like Example 2.5 for any finite Coxeter group.

Remark 2.6 Arbitrary bands (semigroup generated by idempotents) are not weakly ordered in general. Take, for example, $u=x_{1} x_{2}$ and $v=x_{1} x_{2} x_{1}$ in the free band with generators $x_{1}, x_{2}$. Then $u \leq v$ and $v \leq u$, but $u \neq v$.

\section{Radical of weakly ordered semigroup algebras}

We now show that the radical of a weakly ordered semigroup algebra is given by $\operatorname{ker} C$.

Proposition 3.1 Let $S$ be a weakly ordered semigroup and let $R$ be a commutative ring with unity. We extend $C$ linearly to $R S$. Then $\operatorname{ker} C$ is a nilpotent ideal of $R S$. More precisely, $(\operatorname{ker} C)^{n}=0$ where $n$ is length of the longest chain in $(S, \leq)$.

Proof The kernel of $C$ is linearly generated by the elements $v-v^{\prime}$, where $v, v^{\prime} \in S$ are such that $C(v)=C\left(v^{\prime}\right)$.

Let $u \in S$. We prove by downward induction on $\leq$ that there exists a positive integer $n=n_{u}$ such that

$$
u\left(v_{1}-v_{1}^{\prime}\right)\left(v_{2}-v_{2}^{\prime}\right) \cdots\left(v_{n}-v_{n}^{\prime}\right)=0
$$

for all $v_{1}, \ldots, v_{n}, v_{1}^{\prime}, \ldots, v_{n}^{\prime} \in S$ with $C\left(v_{i}\right)=C\left(v_{i}^{\prime}\right)$ for all $1 \leq i \leq n$. The claim follows taking $u$ to be the unity in $S$.

Assume first that $u$ is maximal with respect to $\leq$ in $S$. Then $u=u v$ for all $v \in S$ since $u \leq u v$ by definition of $\leq$. Hence $u\left(v-v^{\prime}\right)=0$ for all $v, v^{\prime} \in S$ (regardless of the values of $C(v)$ and $\left.C\left(v^{\prime}\right)\right)$. So $n_{u}=1$ in this case.

Now suppose we have found $n_{w}$ for all $w \in S$ such that $u<w$. Then take $n=$ $n_{u}=\max \left\{n_{w}+1: u<w\right\}$.

Choose $v_{1}, \ldots, v_{n}, v_{1}^{\prime}, \ldots, v_{n}^{\prime} \in S$ such that $C\left(v_{i}\right)=C\left(v_{i}^{\prime}\right)$ for all $1 \leq i \leq n$. We have $u \leq u v_{1}$. Hence $u=u v_{1}$ or $u<u v_{1}$.

If $u=u v_{1}$, then Lemma 2.3 gives us that $u=u v_{1}^{\prime}$. This means $u\left(v_{1}-v_{1}^{\prime}\right)=0$.

On the other hand, if $u<u v_{1}$, then Lemma 2.3 gives us $u<u v_{1}^{\prime}$. Set $w=u v_{1}$ and $w^{\prime}=u v_{1}^{\prime}$. Then

$u\left(v_{1}-v_{1}^{\prime}\right) \cdots\left(v_{n}-v_{n}^{\prime}\right)=\left(w\left(v_{2}-v_{2}^{\prime}\right) \cdots\left(v_{n}-v_{n}^{\prime}\right)\right)-\left(w^{\prime}\left(v_{2}-v_{2}^{\prime}\right) \cdots\left(v_{n}-v_{n}^{\prime}\right)\right)=0$,

due to our choice of $n$. 
Theorem 3.2 Let $S$ be a weakly ordered semigroup and let $k$ be a field. Then $\operatorname{rad} k S=\operatorname{ker} C$, and the nilpotency index of $\operatorname{rad} k S$ is at most the length of the longest chain in $(S, \leq)$. Moreover all simple representations of $k S$ are of $\operatorname{dim}=1$ and are indexed by the elements of $L$.

Proof Condition (1) implies that $k S / \operatorname{ker} C=k L$. It is well known that $k L$ is a commutative semi-simple algebra (see [1]). Hence $\operatorname{rad} k S \subseteq \operatorname{ker} C$ and all simple representations of $k S$ are of $\operatorname{dim}=1$. Conversely, Proposition 3.1 gives us that $\operatorname{ker} C$ is a nilpotent ideal in $k S$. Hence $\operatorname{ker} C \subseteq \operatorname{rad} k S$ and $L$ indexes the simple representations.

Example 3.3 In the case where $S$ is a left regular band, Theorem 3.2 corresponds to a result of [1]. Brown also computes the minimal idempotents (hence the projective modules) of $k S$.

Example 3.4 To our knowledge, in the case where $S=H_{n+1}(0)$ is a 0-Hecke algebra (as in Example 2.5), Theorem 3.2 gives us the simplest proof of Norton's results [3]. In this case it is an open problem to construct explicitly the minimal idempotents. The best description of the projective modules is given by [2] where they are described explicitly by left ideals $H_{n}(0) v_{I}$ with $I \subseteq\{1,2, \ldots, n\}$. But the elements $v_{I}$ are not the minimal idempotents. In general they are not even idempotents.

Remark 3.5 As mentioned in the foreword, the result presented here is the beginning of a larger project. We are interested in adapting the explicit construction of [1] in order to get the minimal idempotent of a weakly ordered semigroup $S$. The idea would be to lift the idempotents from $L$ using $C$. The hope would be to get a nice description of the minimal idempotents of $H_{n}(0)$.

It would be nice to find other non-trivial examples of weakly ordered semigroups that are not left regular bands or 0-Hecke algebras.

\section{References}

1. Brown, K.S.: Semigroups, rings, and Markov chains. J. Theor. Probab. 13, 871-938 (2000)

2. Krob, D., Thibon, J.-Y.: Noncommutative symmetric functions, IV: quantum linear groups and Hecke algebras at $q=0$. J. Algebr. Comb. 6, 339-376 (1997)

3. Norton, P.N.: 0-Hecke algebras. J. Austral. Math. Soc. Ser. A 27, 337-357 (1978) 